\title{
Habilidades de Resolução Temporal em Músicos Violinistas e Não Músicos
}

\section{Temporal Resolution Abilities in Musicians and No Musicians Violinists}

\author{
Ricardo Alexandre Martinez Monteiro*, Franklin Martins Nascimento*, Carla Debus Soares**, \\ Maria Inês Dornelles da Costa Ferreira***.
}

\author{
* Bacharel em Fonoaudiologia. Fonoaudiólogo. \\ ** Mestrado. Professora do Curso de Fonoaudiologia do Centro Universitário Metodista - IPA (Porto Alegre) e Fonoaudióloga do Setor de Audiologia do Mãe de Deus \\ Center (Porto Alegre). \\ ***: Doutorado. Professora do Curso de Fonoaudiologia do Centro Universitário Metodista - IPA (Porto Alegre) e da Faculdade Nossa Senhora de Fátima (Caxias do Sul). \\ Instituição: Centro Universitário Metodista - IPA \\ Porto Alegre / RS - Brasil. \\ Endereço para correspondência: Maria Inês Dornelles da Costa Ferreira - Rua Luiz Afonso 158 /702 Bairro Cidade Baixa - Porto Alegre / RS - CEP: 90050 -310 - Telefone: \\ (+55 51) 9823-0198 - E-mail: costa.ferreira@terra.com.br \\ Artigo recebido em 5 de Março de 2010. Artigo aprovado em 16 de Maio de 2010.
}

\section{RESUMO}

Introdução:

Objetivo:

Método:

Resultados:

Conclusão:

Palavras-chave:

A resolução temporal é a percepção de um intervalo de tempo em que o indivíduo discrimina dois sons sendo uma habilidade envolvida na música.

Identificar o desempenho da resolução temporal em músicos violinistas e não músicos e correlacionálo à média dos limiares das frequências graves e agudas, bem como, ao tempo de exposição diária à música.

O presente estudo caracterizou-se por ser prospectivo e comparado entre dois grupos, sendo um composto por 20 músicos violinistas e outro por 20 não músicos semi pareados por idade e escolaridade que foram submetidos à avaliação audiológica e ao teste Gaps In Noise (GIN), para avaliar a resolução temporal.

O desempenho do teste GIN do grupo de músicos não foi significativo em relação ao grupo controle seja na orelha direita (OD) ou na esquerda (OE). A correlação entre a média das frequências agudas para OE com o teste GIN foi $(\mathrm{p}=0,001)$ no grupo controle. A média das frequências graves para ambas as orelhas no grupo de músicos foi estatisticamente significativa sendo os maiores valores para OD $(\mathrm{p}=0,001)$.

Não houve diferença entre o desempenho do teste GIN para ambos os grupos assim como a correlação entre o tempo de exposição diária a música e o GIN. O limiar audiométrico das frequências agudas mostrou-se relevante na realização do teste GIN

$\overline{\text { SUMMARY }}$ música, limiar auditivo, percepção auditiva.

Introduction:

The temporal resolution is the perception of a time interval in which the individual discriminates two sounds being a skill involved in music.

Objective:

To identify the performance of temporal resolution in musicians and no musicians violinists and correlate it with the average of the thresholds of low frequencies and acute as well as the time of exposure to music daily.

Method:

This study was characterized by be prospective and compared between two groups, one consisting of 20 violinists and other musicians for 20 semi no musicians matched for age and education were submitted to audiological evaluation and to the test Gaps In Noise (GIN) to evaluate the temporal resolution.

Results:

The test performance of the GIN group of musicians was not significant in the control group is in the right ear (RE) or left (LE). The correlation between the average high frequencies for the LE with the GIN test was $(p=0.001)$ in the control group. The average frequencies for both ears in the group of musicians was statistically significant and the highest values for RE $(p=0.001)$.

Conclusion:

There was no difference between the performance of the GIN test for both groups as well as the correlation between duration of daily exposure to music and GIN. The audiometric threshold of high frequencies seemed to be relevant for testing GIN.

Keywords: 


\section{INTRODUÇÃO}

A intenção em pesquisar o processamento auditivo (central) $(\mathrm{PA}(\mathrm{C})$ ), mais precisamente na habilidade de resolução temporal ocorreu por meio da observação de músicos e da facilidade que eles demonstram em perceber os sons e transformá-los em melodias, tanto sons verbais quanto não verbais. Com isso, pesquisas foram realizadas destacando a relação entre o PA(C) e a música, bem como, a influência de algumas variáveis como o tempo de exposição à música, fatores culturais e familiares (1). O $\mathrm{PA}(\mathrm{C})$ é definido por vários autores como a percepção do som ou da alteração do mesmo dentro de um período restrito de tempo (2). Desenvolve-se desde a infância, sendo que, a exposição ao som aos dois primeiros anos de vida é de extrema importância para o amadurecimento das estruturas do sistema nervoso central (3).

Um estudo aborda a contribuição que o PA(C) pode oferecer para identificar os aspectos relacionados à discriminação auditiva no âmbito escolar (4). Assim, destaca-se a importância do fator tempo para a discriminação dos estímulos acústicos (5,6). O PA(C) pode ser definido como a eficiência e a efetividade com que o sistema nervoso central (SNC) utiliza a informação auditiva. Envolve, além da discriminação auditiva, as habilidades de localização e lateralização do som, reconhecimento, aspectos temporais, testes de escuta dicótica e com sinais acústicos degradados (7). A discriminação auditiva envolve a percepção dos estímulos acústicos em sucessões muito rápidas requerendo precisão das informações que são levadas ao cérebro. Assim, possibilita a decodificação e o entendimento da fala principalmente em situações desfavoráveis, como na presença de ruído de fundo e fala competitiva $(4,8)$. Para a música, a identificação do ritmo depende da percepção do tempo (9).

Dessa forma, o processamento temporal (PT) pode ser definido como a percepção do som ou a alteração do mesmo dentro de um domínio de tempo. O mesmo pode ser observado em muitos níveis do mais básico (regulação de tempo neural no nervo auditivo) até o mais complexo (processamento cortical da audição binaural e da percepção do discurso). Isso permite ao ser humano, a percepção dos sons da fala e compreensão da linguagem oral $(3,10)$. O PT divide-se em quatro subcomponentes: ordenação ou sequencialização temporal, resolução temporal ou discriminação integração temporal ou adição e mascaramento temporal. Neste momento, apenas a resolução temporal será abordada de acordo com o objetivo do presente estudo (10).

A resolução temporal é definida como a percepção de um intervalo curto de tempo em que cada indivíduo pode discriminar entre dois sinais auditivos de aproximadamente 2 a 3 ms. Assim, o limiar de resolução temporal é conhecido como acuidade auditiva temporal ou tempo de integração mínimo (10). Para realizar tal avaliação, Musıek elaborou o teste (GIN), em que o indivíduo deve perceber intervalos de $2 \mathrm{~ms}$ a $20 \mathrm{~ms}$ em meio ao ruído branco, $(8,11$, 12, 13).

Em contrapartida, estudos realizados com músicos apontam que o treinamento musical diário, utilizado por músicos profissionais, pode induzir funcionalmente a reorganização do córtex cerebral. Assim, o contato com a música antes dos sete anos de idade poderia contribuir com o desenvolvimento do $\mathrm{PA}(\mathrm{C})$, e mais precisamente do PT (14). Os músicos pesquisados apresentaram aumento do plano temporal esquerdo identificado através de exames de magnetoencefalografia. Os autores concluíram que os músicos apresentaram melhor ativação neural devido ao treinamento musical de longo prazo (15).

Outra pesquisa ressalta que para haver um melhor desenvolvimento no plano temporal, o estímulo musical deveria iniciar antes dos nove anos de idade sendo importante para o PT. Na comparação de músicos experientes com não músicos, os primeiros responderam diferentemente aos estímulos musicais em comparação com o cérebro dos não músicos. Este fato também foi observado, em músicos que iniciaram a sua atividade musical muito cedo (14). Contudo, outras pesquisas defendem que a habilidade musical é inata e que o treinamento musical não é o responsável pela melhora no PT. No entanto os autores confirmaram a melhora do PT em relação a indivíduos que foram expostos ao estímulo musical precocemente (16). Seguindo o mesmo pressuposto teórico outro estudo aponta que a música tem uma influência positiva no desenvolvimento do PT, pois segundo seu estudo, indivíduos que foram expostos a treinamento musical (cantores) durante quatro anos em comparação a músicos amadores sem orientação profissional, tiveram melhor desempenho na habilidade de resolução temporal através do teste Random Gap Detection Threshold (RGDT), (17). Ao realizar um estudo comparativo entre jovens através dos testes GIN e RGDT, os estudantes de musicoterapia sobressaíram-se em relação às estudantes de fonoaudiologia nos dois testes (12). A música exerce importância no desenvolvimento cerebral e no processamento dos sons sequenciais, no ritmo e na atividade do giro temporal e do lobo frontal demonstrando a existência de uma relação entre o ritmo musical, a fala expressiva e o PT $(18,19)$.

De acordo com o exposto, o objetivo do presente estudo é identificar o desempenho da resolução temporal em músicos violinistas e não músicos. Seguindo estes pressupostos teóricos e a partir das pesquisas realizadas espera-se, através deste trabalho, identificar melhores 
limiares de resolução temporal nos indivíduos músicos. Também objetiva-se a correlação desta variável com a média dos limiares das frequências graves e agudas e média tritonal, bem como, com o tempo de exposição diária à música.

\section{MÉTODO}

O estudo foi realizado no laboratório de audiologia da Instituição, e recebeu a aprovação do Comitê de Ética em Pesquisa, sob o protocolo 123/2008. Caracteriza-se por ser um estudo prospectivo, transversal, individual, observacional, contemporâneo e comparativo.

Após os esclarecimentos dos objetivos da pesquisa os participantes assinaram o Termo de Consentimento Livre Esclarecido, (TCLE) e o responsável pela Instituição da qual pertencia o grupo em estudo assinou o Termo de Conhecimento Institucional (TCI). Os critérios estabelecidos para inclusão dos participantes na pesquisa foram todos os músicos violinistas, que constituíram o grupo em estudo. Para o grupo controle foram incluídos participantes não músicos emparelhados com o grupo em estudo de acordo com a idade. Como critério de exclusão estabeleceu-se aqueles que apresentassem alteração nos limiares auditivos tonais. Como critérios de exclusão para ambos os grupos destacam-se os indivíduos que não aceitaram participar da pesquisa, aqueles que apresentaram timpanometria representativa de alterações na orelha média, bem como os usuários de medicação psicotrópica ou neurológica. Tais critérios foram obtidos por meio de um questionário específico para músicos e não músicos e pelas medidas de imitância acústica. Convém ressaltar que as possíveis alterações na audiometria tonal liminar configuraram critério de exclusão para o grupo de não músicos e variável a ser analisada para o grupo de músicos.

A coleta foi realizada pela manhã para garantir o repouso auditivo no grupo de músicos. Após encaixaremse nos critérios de inclusão todos os participantes realizaram inspeção do meato acústico externo para verificar as condições de realização dos exames subsequentes. As medidas de imitância acústica foram realizadas com o equipamento AT22t da marca interacoustics e constituíram-se de timpanometria e pesquisa dos reflexos acústicos. A audiometria tonal liminar foi o segundo exame realizado através dos equipamentos AC30 e AC33 ambos, da marca Interacoustics. Todos os equipamentos encontraram-se devidamente calibrados na data em que as avaliações foram realizadas.

O terceiro teste utilizado foi o GIN, realizado através da conexão dos audiômetros AC30 ou AC33 com CD player da marca Philips modelo AX2420/78. O teste consiste na detecção de intervalos de silêncio em meio ao ruído branco com a duração de 6 segundos. Tais intervalos variam de 0 a 3 cuja duração varia de 2 a 20 ms, (13). Primeiramente, os participantes realizaram uma prática com a faixa teste após receberem a instrução do mesmo respondendo através da pêra de respostas do audiômetro. Na sequência foi aplicada uma lista para cada orelha a $40 \mathrm{dBNa}$ da média de $500 \mathrm{~Hz}, 1000 \mathrm{~Hz}$ e $2000 \mathrm{~Hz}$, obtida na audiometria tonal liminar. Para descrever o perfil da amostra segundo as variáveis em estudo, foram realizadas tabelas de frequência das variáveis categóricas, com os valores de frequência absoluta (n) e percentual (\%), e estatísticas descritivas das variáveis contínuas, com valores de média, desvio padrão, valores mínimo e máximo, e mediana.

Para comparação das variáveis categóricas entre os grupos foi utilizado o teste exato de Fisher. Para comparar as variáveis numéricas entre dois grupos foi utilizado o teste de Mann-Whitney, e para comparar as variáveis numéricas entre três ou mais grupos foi utilizado o teste de KruskalWallis, devido à ausência de distribuição normal das variáveis. Para comparar as variáveis numéricas entre OD e OE foi utilizado o teste de Wilcoxon para amostras relacionadas, devidoà ausência de distribuição normal das variáveis. Para analisar a relação entre as variáveis numéricas foi utilizado o coeficiente de correlação de Spearman, devido à ausência de distribuição normal das variáveis. O nível de significância adotado para os testes estatísticos foi de $5 \%$, ou seja, $\mathrm{P}<0.05$.

\section{RESULTADOS}

O presente estudo constituiu-se em um grupo de 20 músicos violinistas e outro de 20 participantes não músicos semipareados por idade e escolaridade que formaram o grupo controle, todos do gênero masculino. Em relação à idade participaram do estudo 10 (50\%) músicos com idade inferior a 20 anos, três (15\%) com idade entre 20 e 29 anos de idade e sete (35\%) com idade igual ou superior a 30 anos. Já no grupo controle, nove (45\%) participantes tinham idade inferior a 20 anos, quatro (20\%) com idade entre 20 e 29 anos de idade e sete (35\%) com idade igual ou superior a 30 anos.

Do grupo de músicos, três (15\%) cursaram o ensino fundamental incompleto, seis (30\%) possuíam o ensino médio incompleto, sete (35\%) o ensino completo, dois (10\%) o ensino superior incompleto e dois (10\%) possuíam o ensino superior completo. No grupo controle, por sua vez, cinco (25\%) dos participantes cursavam o ensino fundamental incompleto, três (15\%) possuíam o ensino médio incompleto, sete (35\%) o ensino médio completo, dois (10\%) o ensino superior incompleto e três (15\%) possuíam o ensino superior completo. 
Tabela I. Analise comparativa das variáveis numéricas entre os grupos.

\begin{tabular}{lccccccccccccc}
\hline & \multicolumn{1}{c}{ Não Músicos } & \multicolumn{1}{c}{ Músicos } \\
& $\mathrm{n}$ & M. & D.P. & Min. & Med. & Max. & $\mathrm{n}$ & M. & D.P. & Min. & Med. & Max. & ValorP \\
\hline ldade & 20 & 24,65 & 12,01 & 9,00 & 23,00 & 48,00 & 20 & 24,60 & 12,10 & 10,00 & 22,50 & 48,00 & 0,978 \\
Temp. Aprend. & 00 & - & - & - & - & - & 00 & 7,25 & 7,01 & 1,00 & 5,00 & 25,00 & - \\
Expos. Música & 00 & - & - & - & - & - & 00 & 27,25 & 11,06 & 10,00 & 27,50 & 45,00 & - \\
GIN OD & 20 & 6,00 & 2,13 & 3,00 & 5,00 & 10,0 & 20 & 5,50 & 2,84 & 2,00 & 5,00 & 15,00 & 0,333 \\
GIN OE & 20 & 5,95 & 2,28 & 2,00 & 6,0 & 10,0 & 20 & 4,90 & 1,62 & 2,00 & 5,00 & 10,00 & 0,156 \\
M. Freq. Grav. OD & 20 & 10,45 & 8,20 & $-5,00$ & 11,00 & 25,00 & 20 & 12,80 & 6,89 & $-2,00$ & 12,50 & 23,00 & 0,299 \\
M. Freq. Trit. OD & 20 & 7,05 & 7,58 & $-8,00$ & 7,00 & 20,00 & 20 & 6,85 & 6,68 & $-2,00$ & 6,00 & 27,00 & 0,775 \\
M. Freq. Agud. OD & 20 & 9,25 & 7,40 & $-10,00$ & 9,00 & 25,00 & 20 & 7,30 & 9,20 & $-8,00$ & 6,00 & 35,00 & 0,117 \\
M. Freq. Grav. OE & 20 & 9,25 & 7,11 & $-3,00$ & 8,00 & 22,00 & 20 & 8,55 & 5,84 & $-2,00$ & 8,50 & 20,00 & 0,775 \\
M. Freq. Trit. OE & 20 & 5,90 & 5,44 & $-5,00$ & 6,00 & 15,00 & 20 & 5,05 & 4,54 & $-2,00$ & 5,00 & 12,00 & 0,686 \\
M. Freq. Agud. OE & 20 & 8,50 & 7,29 & $-10,00$ & 8,50 & 25,00 & 20 & 7,35 & 6,66 & $-2,00$ & 8,00 & 27,00 & 0,430 \\
\hline
\end{tabular}

Legenda: Tempo de aprendizagem (Temp. Aprend.); Exposição à música (Expos. Música); Teste GAPS In Noise (GIN), Orelha Direita (OD) e Orelha Esquerda (OE) em músicos violinistas e não-músicos; Media das frequências graves (Med. Freq. Grav.); Média das frequências agudas (Med. Freq. Agud.); Média tritonal (Med. Trit.), em músicos violinistas e não-músicos nas orelhas direita (OD) e orelha esquerda (OE). Média (M); Mínima (Min.), Máxima (Max.); Mediana (Med.).

A Tabela 1 mostra a análise comparativa, das variáveis numéricas entre os grupos. Convém ressaltar que o desempenho do grupo de músicos do teste GIN não foi estatisticamente significativo em relação do grupo controle. No entanto, pode ser verificado maior incidência de alteração para a habilidade de processamento temporal no grupo de não músicos quando comparado ao grupo de músicos. Na sequência, a análise entre os resultados do teste GIN, foram comparadas às variáveis faixa etária e escolaridade em cada grupo, não apresentando relação estatisticamente significativa. A análise de correlação dos resultados obtidos no teste GIN com as variáveis idade, média das frequências graves, média tritonal e média das frequências agudas, por orelha, foram realizadas através do teste de correlação de Spearman considerando os grupos separadamente.

A Figura 1 mostra os resultados do teste GIN obtidos em ambas as orelhas quando correlacionados a variável média das frequências agudas para a OE no grupo controle, sendo a correlação média das frequências agudas para a OE com o desempenho do teste GIN para a OD ( $\mathrm{r}=0,561 \sim$ $\mathrm{p}=0,009)$ e média das frequências agudas para OE com o desempenho do teste GIN para a mesma orelha ( $\mathrm{r}=0,521 \sim$ $p=0,001)$. Tais resultados indicam que quanto mais elevada for a média das frequências agudas da OE, maior será o limiar de detecção de gap no teste GIN no grupo controle.

Verificou-se ainda correlação significativa do tempo de exposição diária à música com o desempenho do teste GIN para a OE, indicando que aumento do tempo de exposição diária à música é diretamente proporcional ao limiar de detecção de gap. Tal resultado pode ser observado na Figura 3. Por fim, a análise comparativa entre as OD e OE foi realizada por grupo, sendo a única variável estatistica-

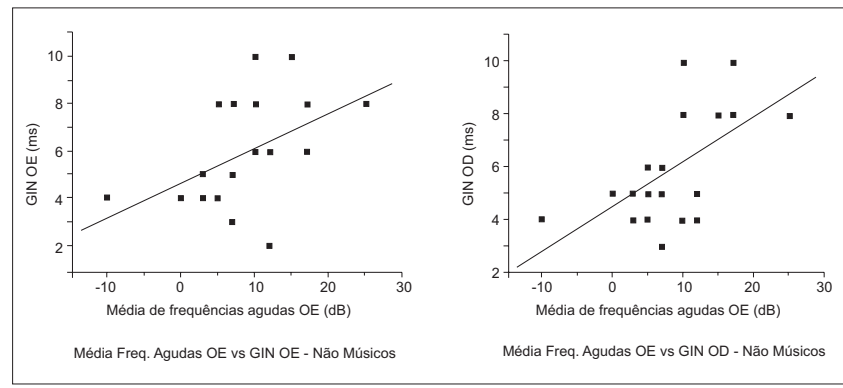

Figura 1. Correlação do teste GIN com a média das frequências agudas no grupo controle da OE e OD. - OD: orelha direita OE: orelha esquerda GIN: Gaps In Noise (intervalo no ruído).

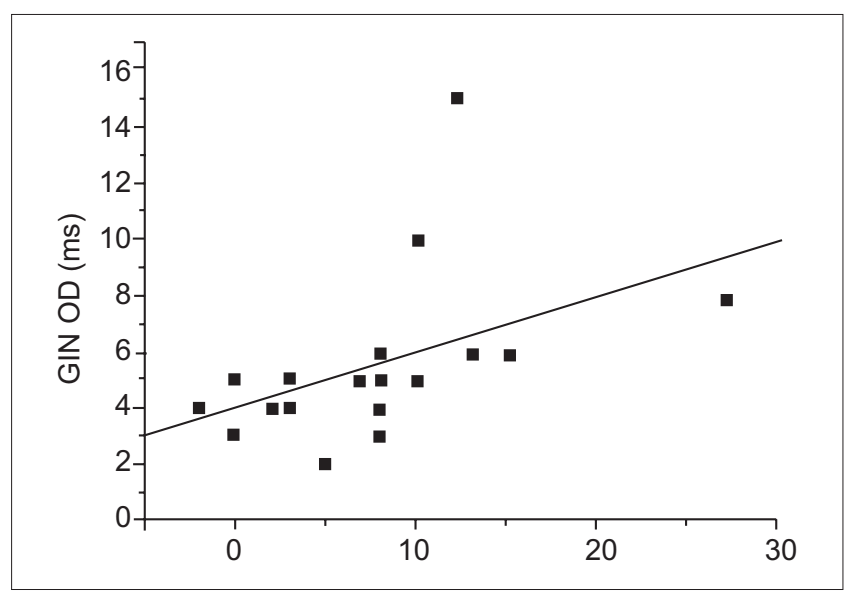

Figura 2. Correlação do teste GIN da OD com a média das frequências agudas da OE no grupo de músicos. - OD: orelha direita OE: orelha esquerda GIN: Gaps In Noise (intervalo no ruído). 


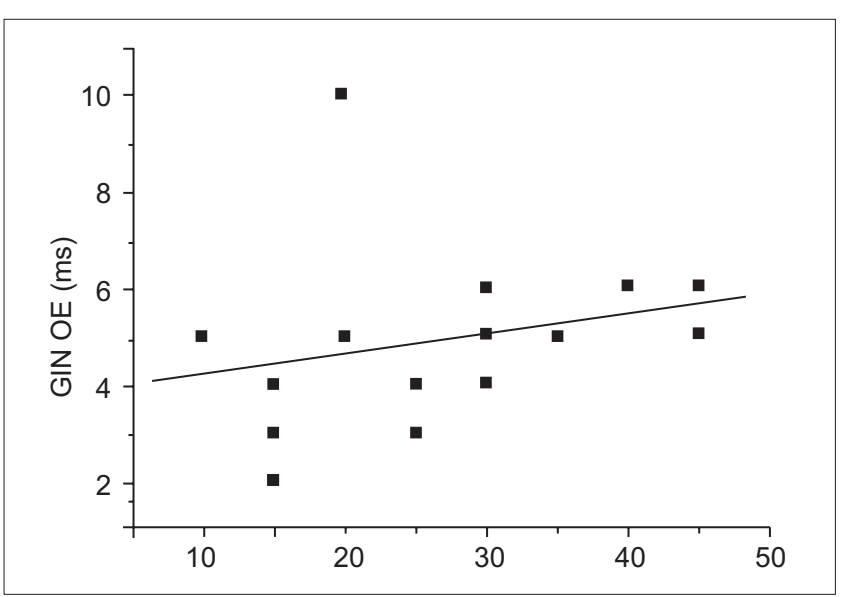

Figura 3. Correlação do teste GIN da OE com o tempo de exposição diária à música. - OE: orelha esquerda GIN: Gaps In Noise (intervalo no ruído).

mente significativa a média das frequências graves comparada entre as orelhas no grupo de músicos sendo que a OD apresentou valores maiores $(p<0,001)$ como mostra a Figura 4. Através deste resultado é possível constatar que o grupo de músicos apresentou limiares audiométricos na OD piores que na orelha contralateral para as frequências graves.

\section{DISCUSSÃO}

A busca de informações sobre a correlação entre música e resolução temporal comparando músicos e não músicos, tem sido um fator frequente nas pesquisas atuais. No teste GIN, aplicado em ambos os grupos, não se obteve relação estatisticamente significativa quanto ao limiar de detecção de intervalo de silêncio. Durante a coleta de dados observou-se que os músicos mais experientes eram hábeis na identificação dos intervalos de gap. Este fato associado ao resultado do presente estudo permitiu atrelar o desempenho da habilidade de resolução temporal a outros fatores como a idade de iniciação musical e o tempo de exposição diária a música.

De acordo com a Tabela 1, o tempo de aprendizado musical variou entre um e 25 anos, com média de 7,25 anos. Quanto à idade, a mínima foi de 10 anos e a máxima de 48, com média de 24,6 anos. Ao considerar a média de idade dos participantes e a média do tempo de aprendizado, estima-se que a idade média de iniciação do aprendizado formal dos músicos foi de 17,4 anos.

Considerando a idade de iniciação musical é responsável pelo desenvolvimento e pela maturação das áreas auditivas relacionadas à música (14), uma pesquisa envol-

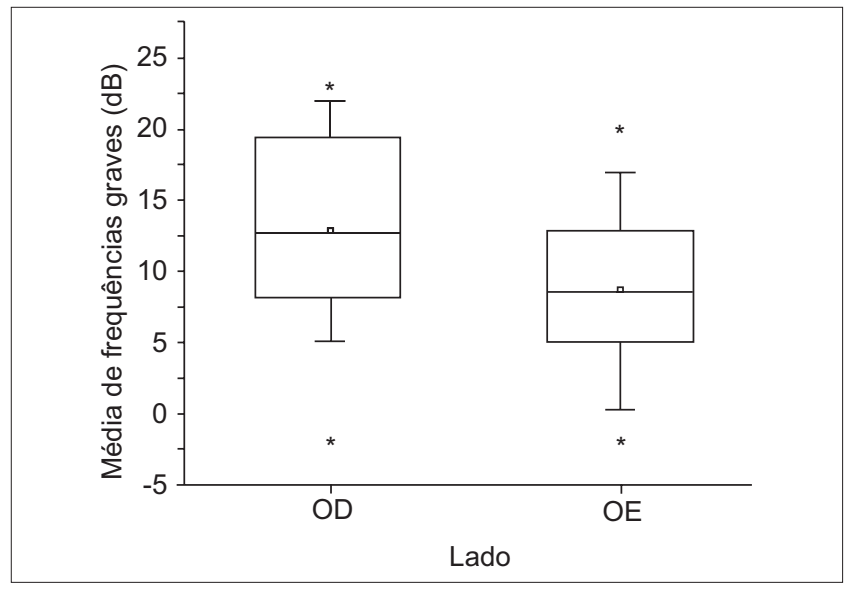

Figura 4. Análise comparativa da média das frequências graves para a OD e OE no grupo de músicos. - OD: orelha direita OE: orelha esquerda.

vendo exames de neuroimagem identificou a predominância hemisférica esquerda no desempenho das habilidades de PT e concluiu que o pitch e a detecção de intervalos de tempo mostraram-se rebaixadas em indivíduos com lesão hemisférica esquerda (19). Outros estudos também consideraram o tempo de iniciação e/ou treinamento musical como relevante para o desempenho como as habilidades relacionadas à percepção do tempo $(17,20)$.

O tamanho da população e a possível existência de músicos com alteração da habilidade de resolução temporal também são fatores a serem considerados. No grupo de músicos, o limiar de detecção de gap máximo para a OD foi de $15 \mathrm{~ms}$ ao passo que no grupo de não músicos a mesma medida foi de $10 \mathrm{~ms}$. A figura 1 mostra a correlação do teste GIN com a média das frequências agudas para o grupo de não músicos, ou seja, o aumento do limiar para as frequências agudas é diretamente proporcional ao limiar de deteç̧ão de gap. Convém ressaltar que o referido grupo apresentou uma média de 9,25 dB para as frequências agudas da OD e 8,50 dB para a mesma faixa de frequência da OE, sendo estes, valores situados no padrão de normalidade. Assim, destaca-se a relevância dos limiares das frequências agudas no desempenho do teste GIN. A referida faixa de frequência possui ondas de menor comprimento cujas células responsáveis pela identificação dos intervalos de tempo devem se encontrar intactas. As frequências agudas também são mais eficientes para a detecção de gap devido ao uso de filtros de banda larga, para a percepção e constatado que quanto mais larga a banda, melhor o desempenho da resolução temporal (2, 8). Dessa forma, a elevação do limiar audiométrico das frequências altas pode influenciar nos resultados do teste GIN (2). 
Os resultados evidenciados na Figura 2 mostram a correlação estatisticamente significativa do teste GIN da OD com a média das frequências agudas da OE no grupo de músicos. Tal constatação evidencia resultados semelhantes entre o grupo de músicos e não-músicos em relação à relevância dos limiares das frequências agudas no desempenho do teste GIN (2), seja na orelha homolateral como na contralateral, porém ainda não é possível explicar tal relação.

A Figura 3 também mostra a relação estatisticamente significativa entre o tempo de exposição diária à música e o desempenho do teste GIN da OE, ou seja, o aumento da primeira variável é diretamente proporcional ao aumento da segunda que representa a piora no desempenho da resolução temporal. Dessa forma, é possível discutir que o tempo de exposição diária a música não se constitui como facilitador do desempenho em resolução temporal, talvez, em função da iniciação musical tardia (14)

A Figura 4 apresentou a análise comparativa da média das frequências graves entre as orelhas no grupo de músicos apontando que os limiares da OD do referido grupo foram piores quando comparados aos limiares da OE. Os músicos pesquisados, expõe a OD a outros instrumentos, bem como a outros violinos, a um nível de pressão sonora elevado que poderia justificar o aumento do limiar. Um estudo pesquisou 30 músicos expostos a níveis de pressão sonora superiores a 85 dBNPS e comprovou alteração nos limiares auditivos em ambas as orelhas (21). Por outro lado, uma pesquisa com trabalhadores expostos a ruído ocupacional também identificou piores limiares na OD sem motivo aparente (22).

Num estudo envolvendo músicos da orquestra sinfônica de Minas Gerais identificou-se maior perda auditiva na OE nas frequências agudas e não na média de frequências graves como nos achados deste estudo (23). Convém ressaltar que o violino, produz ondas originadas pela vibração das cordas em atrito, que se propagam através do seu corpo fornecendo uma intensidade perto de $80 \mathrm{dBNPS}$ cuja propagação resulta em ressonâncias direcionais para as frequências altas, relacionando-se, assim, com o resultado do presente estudo (24).

\section{CONCLUSÃO}

O desempenho do teste GIN não foi estatisticamente significativo em relação ao grupo controle. O limiar das frequências agudas mostrou-se relevante na determinação do limiar de detecção de gap para ambos os grupos. Além, disso o tempo de exposição diária à música não se constituiu como facilitador do desempenho da resolução temporal. Outros estudos com uma população maior e com músicos cuja iniciação musical tenha ocorrido mais cedo se tornam necessários.

\section{REFERÊNCIAS BIBLIOGRÁFICAS}

1. Escalda J. Educação musical e suas relações com habilidades auditivas e o desenvolvimento fonológico de crianças de três e seis anos. Anais do IV Simpósio de Cognição e Artes Musicais (SIMCAM), 2008.

2. Samelli AG, SchochatE. Processamento auditivo resolução temporal e teste de detecção de Gap: revisão de literatura. Rev Cefac. 2008, 10(3):369-377

3. Costa-Ferreira MID. A influência da terapia do processamento auditivo na compreensão em leitura: Uma abordagem conexionista. Porto Alegre, 2007, p. 165, (Tese de Doutorado) - Faculdade de Letras - Programa de pósgraduação em letras. PUCRS.

4. Neves IF, Schochat E. Maturação do processamento auditivo em crianças com e sem dificuldades escolares. PróFono. 2005, 17(3):311-320.

5. Neves VT, Feitosa MA. Controvérsias na relação entre processamento temporal auditivo e envelhecimento. Rev Bras Otorrinolaringol. 2003, 69(2):242-249

6. Rabelo CM. Avaliação eletrofisiológica e comportamental do processamento temporal. São Paulo, 2008, p.181, (Tese de Doutorado) - Faculdade de Medicina. Universidade de São Paulo.

7. ASHA: American Speech-Language-Hearing Association. (Central) Auditory Processing Disorders[Technical Report], 2005 Disponível em: www.asha.org/policy.

8. Samelli AG, Schochat E. The gaps-in-noise test: gap detection thresholds in normal-hearing young adults. Int J Audiol. 2008, 47(5):238-245.

9. Boker SM. The perception of structure in simple auditory rhythmic patterns. Virginia, 1994. p. 112 (Master of Arts) University of Virginia: Department of Psychology.

10. Shinn JB. Temporal processing and temporal patterning tests. In: Musiek FE, Charmak GD. Handbook of (central) auditory processing disorders: auditory neuroscience and diagnosis. San Diego: Plural Publishing; 2007, Vol. 1, pp 231-243.

11. Musiek FE. Gaps In Noise (GIN test) Full version, Storrs: Audiology Illustrate, 2003. 
12. Zaidan E, Garcia AP, Tedesco MLF, Baran JA. Desempenho de adultos jovens normais em dois testes de resolução temporal. Pró-Fono. 2008, 20(1):19-24.

13. Musiek FE, Shinn JB, Jirsa R, Bamiou DE, Baran J, Zaidan E. Gin (Gaps-In-Noise) test performance in subjects with confirmed central auditory nervous system involvement. Ear Hear. 2005, 26(6):608-618.

14. Ohnishi T, Matsuda H, Asada T, Aruga M, Hirakata M, Nishikawa M. Functional anatomy of musical perception in musicians. Cerebr Cortex. 2001, 11(8):754-760.

15. Schalaug G. The brain of musicians: a model for functional and structural adaptation. Ann New York Acad Sci. 2001, 930: 281-299.

16. Pantev C, Roberts LE, Schulz M, Engelien A, Ross B. Timbre specific enhancement of auditory cortical representations in musicians. NeuroReport. 2001, 12(1): 169-174.

17. Ishll C, Arashiro PM, Pereira LD. Ordenação e resolução temporal em cantores profissionais e amadores afinados e desafinados. Pró-Fono. 2006, 18(3):285-292.
18. Muszkat M, Correia CMF, Campos SM. Música e neurociências. Rev Neurociências. 2000, 8(2):70-75.

19. Andrade PE. Uma abordagem evolucionária e neurocientífica da música. Neurociencias. 2004, 1(1):21-33.

20. Rammsayer T, Altenmüller E. Temporal information processing in musicians and non-musicians. Music Perception. 2006, 24(1):37-48.

21. Amorim RB, Lopes AC, Santos KTP, Melo ADP, Lauris JRP. Alterações Auditivas da Exposição Ocupacional em Músicos. Arq Int Otorrinolaringol. 2008, 12(3):377-383.

22. Santos JD, Costa-Ferreira MID. Variação dos limiares audiométricos em trabalhadores submetidos a Ruído ocupacional, Arq Int Otorrinolaringol. 2008, 12(2):201-209.

23. Maia AA, Gonçalves DU, Menezes LN, Barbosa BM, Almeida PS, Resende LM. Análise do perfil audiológico dos músicos da orquestra sinfônica de Minas Gerais (OSMG). Per Musi. 2007,15:67-71.

24. Donoso JP, Tannús A, Guimarães F, Freitas TC. A física do violino. Rev Bras Ensino Fis. 2008, 30(2):2305.1-2305.21. 